\title{
Ramsey numbers of ordered graphs
}

\author{
Martin Balko ${ }^{1,2}$ Josef Cibulka $^{1,3}$ Karel Král ${ }^{1,4}$
}

Department of Applied Mathematics

Charles University, Faculty of Mathematics and Physics

Malostranské nám. 25, 11800 Praha 1, Czech Republic

\author{
Jan Kynčl ${ }^{1,5}$
}

Department of Applied Mathematics and Institute for Theoretical Computer Science

Charles University, Faculty of Mathematics and Physics

Malostranské nám. 25, 11800 Praha 1, Czech Republic

Alfréd Rényi Institute of Mathematics

Reáltanoda u. 13-15, Budapest 1053, Hungary

École Polytechnique Fédérale de Lausanne, Chair of Combinatorial Geometry

EPFL-SB-MATHGEOM-DCG, Station 8, CH-1015 Lausanne, Switzerland

\begin{abstract}
An ordered graph is a graph together with a total ordering of its vertices. We study ordered Ramsey numbers, the analogue of Ramsey numbers for ordered graphs.

In contrast with the case of unordered graphs, we show that there are ordered matchings whose ordered Ramsey numbers are super-polynomial in the number of vertices.

We also prove that ordered Ramsey numbers are polynomial in the number of vertices of the given ordered graph $\mathcal{G}$ if $\mathcal{G}$ has constant degeneracy and constant interval chromatic number or if $\mathcal{G}$ has constant bandwidth. The latter result answers positively a question of Conlon, Fox, Lee, and Sudakov.
\end{abstract}


For a few special classes of ordered graphs, we give asymptotically tight bounds for their ordered Ramsey numbers. For so-called monotone cycles we compute their ordered Ramsey numbers exactly.

Keywords: ordered graph, Ramsey number, ordered Ramsey number

\section{Introduction}

Ramsey's theorem states that for every graph $G$, there exists a number $N$ such that every 2-coloring of the edges of $K_{N}$ contains a monochromatic copy of $G$. The least such $N$ is called the Ramsey number of $G$ and we denote it by $\mathrm{R}(G)$.

In this paper, we study an analogue of Ramsey's theorem for graphs with ordered vertex sets. In particular, we focus on the effects of different vertex orderings on the Ramsey numbers of given graphs.

An ordered graph $\mathcal{G}$ is a pair $(G, \prec)$ where $G$ is a graph and $\prec$ is a total ordering of its vertex set. We say that two ordered graphs $\left(G, \prec_{1}\right)$ and $\left(H, \prec_{2}\right)$ are isomorphic if $G$ and $H$ are isomorphic via a one-to-one correspondence $g: V(G) \rightarrow V(H)$ that also preserves the orderings $\prec_{1}$ and $\prec_{2}$. Note that there is only one ordered complete graph $\mathcal{K}_{n}$ up to isomorphism. An ordered graph $\left(H, \prec_{1}\right)$ is an ordered subgraph of $\left(G, \prec_{2}\right)$ if $H$ is a subgraph of $G$ and $\prec_{1}$ is a suborder of $\prec_{2}$.

For an ordered graph $\mathcal{G}$, we denote by $\overline{\mathrm{R}}(\mathcal{G})$ the smallest number $N$ such that every 2-coloring of the edges of $\mathcal{K}_{N}$ contains a monochromatic copy of $\mathcal{G}$ as an ordered subgraph. The number $\overline{\mathrm{R}}(\mathcal{G})$ is called the ordered Ramsey number of $\mathcal{G}$. Since $\mathcal{K}_{n}$ is unique up to isomorphism, for every ordered graph $\mathcal{G}=(G, \prec)$ with $n$ vertices we have $\mathrm{R}(G) \leq \overline{\mathrm{R}}(\mathcal{G}) \leq \mathrm{R}\left(K_{n}\right)$.

1 The first and the fourth author were supported by the grants GAUK 1262213 of the Grant Agency of Charles University and by the project CE-ITI (GAČR P202/12/G061) of the Czech Science Foundation. The first author was partially supported by the Charles University Grant SVV-2015-260223. The third author acknowledges the support from the European Research Council under the European Union's Seventh Framework Programme (FP/2007-2013) / ERC Grant Agreement n. 616787. The fourth author was also supported by ERC Advanced Research Grant no 267165 (DISCONV) and by Swiss National Science Foundation Grants 200021-137574 and 200020-14453. Part of the research was conducted during the DIMACS REU 2013 program.

2 Email: balko@kam.mff.cuni.cz

3 Email: cibulka@kam.mff.cuni.cz

4 Email: kralka@kam.mff.cuni.cz

5 Email: kyncl@kam.mff.cuni.cz 
Classical results of Erdős [6] and Erdös and Szekeres [7] give the exponential bounds $2^{n / 2} \leq \mathrm{R}\left(K_{n}\right) \leq 2^{2 n}$. Thus the ordered Ramsey numbers are well-defined and are at most exponential in the number of vertices of the given ordered graph.

The concept of ordered graphs appeared earlier in the literature, but we are not aware of any earlier Ramsey-type results for such graphs except for the case of monotone paths and hyperpaths $[2,5,8,11,12]$. During the preparation of this paper, we learned that ordered Ramsey numbers were independently studied by Conlon, Fox, Lee, and Sudakov [4]. There are overlaps between many of our results. We mention these overlaps explicitly in the paper.

The Ramsey type problems for ordered (hyper)graphs appear in many examples such as the Erdös-Szekeres lemma on monotone subsequences [7], the Happy ending theorem [7], geometric Ramsey numbers $[9,10]$, and there are also connections with the extremal theory of $\{0,1\}$-matrices.

Due to space restrictions, all the proofs, as well as some results, have been omitted; the reader can find them in the preliminary full version [1] of the paper.

\section{Specific classes of ordered graphs}

Here we provide several examples of ordered graphs and their ordered Ramsey numbers. It follows from these examples that the ordered Ramsey numbers can be asymptotically different for two orderings of the same graph.

A monotone path $\left(P_{n}, \prec_{m o n}\right)$ is the ordering of the path $P_{n}$ with edges between vertices that are consecutive in $\prec_{\text {mon }}$, see part a) of Figure 1. A straightforward generalization of the proof of the Erdős-Szekeres lemma on monotone subsequences [7] gives $\overline{\mathrm{R}}\left(\left(P_{n}, \prec_{\text {mon }}\right)\right)=(n-1)^{2}+1$. This result can be generalized even to the multi-colored case $[2,11]$.

On the other hand, there is an ordering of the path $P_{n}$ for which the corresponding ordered Ramsey number is linear in $n$. This ordering, called an alternating path, is sketched in part b) of Figure 1. Details can be found in $[1]$.

Choudum and Ponnusamy [2] showed a recursive formula which determines the value of $\overline{\mathrm{R}}\left(\mathcal{S}_{1}, \mathcal{S}_{2}\right)$ exactly for every pair of ordered stars $\mathcal{S}_{1}=\left(K_{1, n_{1}-1}, \prec_{1}\right)$ and $\mathcal{S}_{2}=\left(K_{1, n_{2}-1}, \prec_{2}\right)$. Their result implies that $\overline{\mathrm{R}}\left(\mathcal{S}_{1}, \mathcal{S}_{2}\right)$ is linear in $n_{1}+n_{2}$. Again, see [1] for more details. Here, for ordered graphs $\mathcal{G}$ and $\mathcal{H}$, we use $\overline{\mathrm{R}}(\mathcal{G}, \mathcal{H})$ to denote the least number $N$ such that every red-blue coloring of the edges of $\mathcal{K}_{N}$ contains either a red copy of $\mathcal{G}$ or a blue copy of $\mathcal{H}$. 


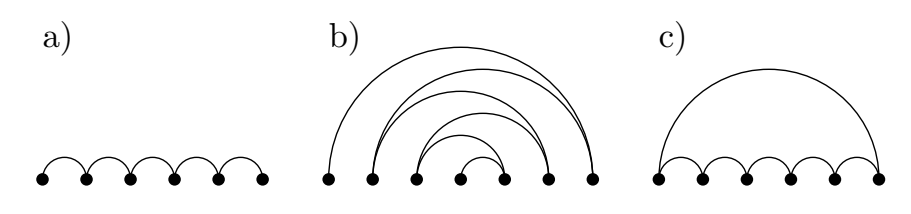

Fig. 1. Examples of ordered graphs: a) a monotone path, b) an alternating path, c) a monotone cycle.

Another example of ordered graphs whose ordered Ramsey numbers are known exactly are monotone cycles. A monotone cycle $\left(C_{n}, \prec_{\text {mon }}\right)$ consists of a monotone path with an additional edge between the first and the last vertex in $\prec_{\text {mon }}$, see part c) of Figure 1. In [1] we prove the following.

Theorem 2.1 For all integers $r \geq 2$ and $s \geq 2$ we have

$$
\overline{\mathrm{R}}\left(\left(C_{r}, \triangleleft_{m o n}\right),\left(C_{s}, \triangleleft_{m o n}\right)\right)=2 r s-3 r-3 s+6 .
$$

As a corollary of Theorem 2.1 we obtain tight bounds for so-called geometric and convex geometric Ramsey numbers of cycles introduced by Károlyi et al. $[9,10]$.

\section{$3 \quad$ Sparse ordered graphs}

In the unordered case, Chvátal et al. [3] showed that for every positive integer $\Delta$ there exists a constant $C(\Delta)$ such that every graph $G$ with $n$ vertices and maximum degree $\Delta$ satisfies $\mathrm{R}(G) \leq C(\Delta) n$. That is, (unordered) Ramsey numbers of graphs with bounded maximum degree are linear in the number of vertices. In a sharp contrast to this fact, we have the following result.

Theorem 3.1 There are arbitrarily large ordered matchings $\mathcal{M}_{n}$ on $n$ vertices such that

$$
\overline{\mathrm{R}}\left(\mathcal{M}_{n}\right) \geq n^{\frac{\log n}{5 \log \log n}}
$$

In other words, the ordered Ramsey numbers can grow super-polynomially in the number of vertices even when we restrict ourselves to ordered graphs with maximum degree 1 . In fact, Conlon et al. [4] proved that as $n$ goes to infinity, almost every ordering $\mathcal{M}_{n}$ of a matching on $n$ vertices satisfies $\overline{\mathrm{R}}\left(\mathcal{M}_{n}\right) \geq n^{\frac{\log n}{20 \log \log n}}$. It follows from both of these results that ordered Ramsey numbers of the same graph can grow super-polynomially in the size of the graph in one ordering and remain linear in another ordering.

For an ordered graph $\mathcal{G}$, the interval chromatic number of $\mathcal{G}$ is the minimum number of intervals the vertex set of $\mathcal{G}$ can be partitioned into such that there 
is no edge between vertices of the same interval. In the following theorem we give a polynomial upper bound for ordered Ramsey numbers of ordered graphs for which the degeneracy as well as the interval chromatic number are both constant.

Theorem 3.2 For positive integers $k$ and $p$, every $k$-degenerate ordered graph $\mathcal{G}$ with $n$ vertices and interval chromatic number $p$ satisfies

$$
\overline{\mathrm{R}}(\mathcal{G}) \leq n^{(1+2 / k)(k+1)^{\left\lceil\log _{2} p\right\rceil}-2 / k} .
$$

This implies that there are orderings under which the ordered Ramsey number of a $k$-degenerate graph with $n$ vertices is polynomial in $n$. Independently, Conlon et al. [4] showed that there exists a constant $c$ such that every $n$-vertex ordered graph $\mathcal{G}$ with degeneracy $k$ and interval chromatic number $p$ satisfies $\overline{\mathrm{R}}(\mathcal{G}) \leq n^{c k \log p}$. This gives a stronger estimate than Theorem 3.2.

We also give a polynomial upper bound on ordered Ramsey numbers of ordered graphs that admit the following recursive decomposition.

For given integers $k, q \geq 2$, we say that an ordered graph $\mathcal{G}=(G, \prec)$ is $(k, q)$-decomposable if $\mathcal{G}$ has at most $k$ vertices or if $\mathcal{G}$ satisfies the following: there is a nonempty interval $I$ with at most $k$ vertices of $\mathcal{G}$ such that the interval $I_{L}$ of vertices of $\mathcal{G}$ that are to the left of $I$ and the interval $I_{R}$ of vertices of $\mathcal{G}$ that are to the right of $I$ satisfy $\left|I_{L}\right|,\left|I_{R}\right| \leq|V(G)| \cdot \frac{q-1}{q}$ and there is no edge between $I_{L}$ and $I_{R}$. Moreover, the ordered graphs $\left(G\left[I_{L}\right], \prec \uparrow_{I_{L}}\right)$ and $\left(G\left[I_{R}\right], \prec \uparrow_{I_{R}}\right)$ are $(k, q)$-decomposable.

Theorem 3.3 For every $k \geq 1$, there is a constant $c_{k}$ such that for every $q \geq 2$ and every $(k, q)$-decomposable ordered graph $\mathcal{G}$ we have

$$
\overline{\mathrm{R}}(\mathcal{G}) \leq 2^{c_{k}\left(\left\lceil\log _{q /(q-1)} n\right\rceil\right.} .
$$

An ordered graph $(G, \prec)$ has bandwidth at most $k$ if we have $|i-j| \leq k$ for every edge $\{u, v\}$ of $G$ where $u$ is the $i$ th and $v$ is the $j$ th vertex in $\prec$. Note that every ordered graph with bandwidth at most $k$ is $(k, 2)$-decomposable. Therefore, Theorem 3.3 implies that for every positive integer $k$ there exists a constant $c_{k}$ such that $\overline{\mathrm{R}}(\mathcal{G} ; 2) \leq n^{c_{k}}$ for every ordered graph $\mathcal{G}$ on $n$ vertices with bandwidth at most $k$. This solves a problem asked by Conlon et al. [4, Problem 6.9].

We end with two open questions concerning ordered Ramsey numbers of sparse ordered graphs. First, for some fixed constant $\Delta$, is there a sequence $\left(G_{n}\right)_{n}$ of $\Delta$-regular graphs $G_{n}$ on $n$ vertices such that $\min _{\prec}\left\{\overline{\mathrm{R}}\left(\left(G_{n}, \prec\right)\right)\right\}$ is superlinear in $n$ where the minimum is taken over all vertex orderings $\prec$ of 
$G_{n}$ ? Second, is there an absolute constant $c>0$ such that for every fixed $\Delta$ there is a sequence $\left(\mathcal{G}_{n}\right)_{n}$ of ordered $\Delta$-regular graphs $\mathcal{G}_{n}$ on $n$ vertices with interval chromatic number 2 such that $\bar{R}\left(\mathcal{G}_{n}\right) \geq n^{c \Delta}$ ? More open questions can be found in [4].

\section{References}

[1] Balko, M., J. Cibulka, K. Král, and J. Kynčl, Ramsey numbers of ordered graphs, URL: http://arxiv.org/abs/1310.7208, 2014.

[2] Choudum, S.A., and B. Ponnusamy, Ordered Ramsey numbers, Discrete Math. 247(1-3) (2002), 79-92.

[3] Chvátal, V., V. Rödl, E. Szemerédi, and W.T. Trotter Jr., The Ramsey number of a graph with bounded maximum degree, J. Combin. Theory Ser. B 34 (1983), 239-243.

[4] Conlon, D., J. Fox, C. Lee, and B. Sudakov, Ordered Ramsey numbers, URL: http://arxiv.org/abs/1410.5292, 2014.

[5] Eliáš, M., and J. Matoušek, Higher-order Erdős-Szekeres theorems, Adv. Math. 244 (2013), 1-15.

[6] Erdős, P., Some remarks on the theory of graphs, Bull. Amer. Math. Soc. 53(4) (1947), 292-294.

[7] Erdős, P., and G. Szekeres, A combinatorial problem in geometry, Compos. Math. 2 (1935), 463-470.

[8] Fox, J., J. Pach, B. Sudakov, and A. Suk, Erdös-Szekeres-type theorems for monotone paths and convex bodies, Proc. London Math. Soc. 105 (2012), 953982.

[9] Károlyi, G., J. Pach, and G. Tóth, Ramsey-type results for geometric graphs, I, Discrete Comput. Geom. 18(3) (1997), 247-255.

[10] Károlyi, G., J. Pach, G. Tóth, and P. Valtr, Ramsey-type results for geometric graphs, II, Discrete Comput. Geom. 20(3) (1998), 375-388.

[11] Milans, K.G., D. Stolee, and D.B. West, Ordered Ramsey theory and track representations of graphs, preprint, URL: http://www.math.illinois.edu/ dwest/pubs/ordram.pdf, 2014.

[12] Moshkovitz, G., and A. Shapira, Ramsey theory, integer partitions and a new proof of the Erdös-Szekeres Theorem, Adv. in Math. 262 (2014), 1107-1129. 